\title{
1 Cooperative Effects of RIG-I-like Receptor Signaling and IRF1 on DNA Damage-Induced
}

\section{Cell Death}

3

4 David Y. Zander ${ }^{1,2}$, Sandy S. Burkart ${ }^{1,3}$, Sandra Wüst ${ }^{1}$, Vladimir G. Magalhães ${ }^{1}$, Marco Binder ${ }^{1, \S}$ 5

6

$7 \quad{ }^{1}$ Research Group "Dynamics of Early Viral Infection and the Innate Antiviral Response", Division

8 Virus-Associated Carcinogenesis (F170), German Cancer Research Center, Heidelberg, Germany

$9 \quad{ }^{2}$ Department of Infectious Diseases, Molecular Virology, Centre for Integrative Infectious Disease

10 Research, Heidelberg University, Heidelberg, Germany

$11{ }^{3}$ Faculty of Biosciences, Heidelberg University, Heidelberg, Germany

12

$13 \quad{ }^{\S}$ Corresponding author: m.binder@dkfz.de 


\section{$\underline{\text { Abstract }}$}

Properly responding to DNA damage is vital for eukaryotic cells, including the induction of DNA repair, growth arrest and, as a last resort to prevent neoplastic transformation, cell death. Besides being crucial for ensuring homeostasis, the same pathways and mechanisms are at the basis of chemoradiotherapy in cancer treatment, which involves therapeutic induction of DNA damage by chemical or physical (radiological) measures. Apart from typical DNA damage response mediators, the relevance of cellintrinsic antiviral signaling pathways in response to DNA breaks has recently emerged. Originally known for combatting viruses via expression of antiviral factors including IFNs and establishing of an antiviral state, RIG-I-like receptors (RLRs) were found to be critical for adequate induction of cell death upon the introduction of DNA double-strand breaks. We here show that presence of IRF3 is crucial in this process, most likely through direct activation of pro-apoptotic factors rather than transcriptional induction of canonical downstream components, such as IFNs. Investigating genes reported to be involved in both DNA damage response and antiviral signaling, we demonstrate that IRF1 is an obligatory factor for DNA damage-induced cell death. Interestingly, its regulation does not require activation of RLR signaling, but rather sensing of DNA double strand breaks by ATM and ATR. Hence, even though independently regulated, both RLR signaling and IRF1 are essential for proper induction/execution of intrinsic apoptosis. Our results not only support more broadly developing IRF1 as a biomarker predictive for the effectiveness of chemoradiotherapy, but also suggest investigating a combined pharmacological stimulation of RLR and IRF1 signaling as a potential adjuvant regimen in tumor therapy.

\section{Introduction}

DNA damage is a ubiquitous and existential threat to organisms. Potential causes comprise ionizing radiation (IR), genotoxic chemicals, but also cell-intrinsic mechanisms. Among various possible DNA alterations, the most drastic and impactful are DNA double-strand breaks (DSBs). Complex mechanisms involving detection by ATM, ATR, and downstream processes including the tumor suppressor p53 and checkpoint inhibition, either lead to sufficient repair of the damage or to induction of programmed cell death $[1,2]$. The latter mostly comprises apoptosis, but other forms such as necroptosis and pyroptosis have recently been reported as well. Mutations of the central DSB sensors can cause severe diseases such as ataxia telangiectasia, associated with carcinogenesis and serious immunodeficiency [3-5]. Originally discovered and best-studied in the context of the antiviral innate immune response, IRF1 has been implicated in the DNA damage response and tumor suppressor functions [6-9].

Following the IRF1 example, it became apparent that cell-intrinsic antiviral signaling pathways also substantially contribute to DNA damage-induced cell death. Both STING and RIG-I-like receptor (RLR) pathways detect damage-associated molecular patterns (DAMPs), such as endogenous DNA 
shown to induce death of breast cancer cells, putting forward a potential application in tumor therapy [12]. Typically, the RLRs, RIG-I and MDA5, are stimulated by non-self RNA in the event of viral infection. Interaction with their adaptor MAVS leads to activation of the transcription factors IRF3, NF$\kappa \mathrm{B}$ p65/RELA and p50/NFKB1. The resulting expression of ISGs and IFNs of type I/III causes the establishment of an antiviral state and, in most cases, effective containment of the invading pathogen. In addition to apoptosis sensitizing effects of NF-KB and IFNs through expression of pro-apoptotic factors, direct cell death mediating effects have recently been reported for MAVS and IRF3 [13, 14]. Chattopadhyay et al. were first to identify and characterize the RLR-induced IRF3-mediated pathway of apoptosis (RIPA) [15]. Stimulation of RLRs with dsRNA or viral infection induces MAVSdependent ubiquitination of IRF3 and subsequent activation of pro-apoptotic factors independent of IRF3's transcriptional activity [16]. Furthermore, MAVS was shown to directly interact with procaspase-8, forming so-called MAVS-death-inducing signaling complexes upon viral infection [17]. Here we show that RLR signaling, IRF1, and canonical DNA damage response pathways, comprising ATM/ATR and p53, are essential for efficient induction of apoptosis. We show that these pathways have independent pro-apoptotic capacities, and we present new insights into IRF1's complex cellular functions.

\section{Methods}

Cell culture, cell line generation, and stimulation. Cell lines were grown at $37{ }^{\circ} \mathrm{C}, 95 \%$ humidity, and $5 \% \mathrm{CO}_{2}$ in Dulbecco's modified eagle medium (DMEM high glucose, Life Technologies, Carlsbad, CA, USA), supplemented with final $10 \%(\mathrm{v} / \mathrm{v})$ fetal calf serum (FCS, Thermo Fisher Scientific, Waltham, MA, USA), 1x non-essential amino acids (Thermo Fisher Scientific), and $100 \mathrm{U} / \mathrm{ml}$ penicillin and $100 \mathrm{ng} / \mathrm{ml}$ streptomycin (LifeTechnologies). For generation of transgene expressing A549 cell lines by lentiviral transduction, lentiviral particles were produced by transfecting HEK 293T cells with plasmids pCMV-dr8.91, pMD2.G, and the respective retroviral vector (pWPI) using calcium phosphate transfection (CalPhos Mammalian Transfection Kit, Takara Bio Europe, SaintGermain-en-Laye, France). After two days the supernatant was harvested, sterile filtered, and used to transduce target cells two times for $24 \mathrm{~h}$. Transduced cells were selected with antibiotics appropriate for the encoded resistance gene (5 $\mu \mathrm{g} / \mathrm{ml}$ blasticidin, MP Biomedicals, Santa Ana, CA, USA; $1 \mu \mathrm{g} / \mathrm{ml}$ puromycin, Sigma Aldrich; 1 mg/ml geneticin (G418), Santa Cruz, Dallas, TX, USA). Knockout (KO) cell lines were generated by clustered regularly interspaced short palindromic repeats (CRISPR)/Cas9 technology. DNA oligonucleotides coding for guideRNAs against the respective genes (sequences shown in Supplementary Table S1) were cloned into the expression vector LentiCRISPRv2 (Feng Zhang, Addgene \#52961).

Transduced A549 wild-type cells were selected with puromycin, single cell clones were isolated, and

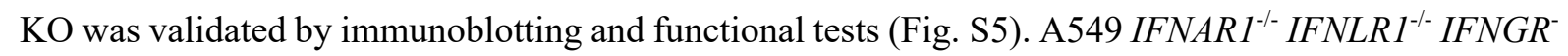

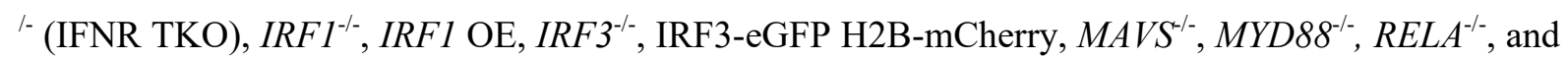


$R I G-I^{-/}$were reported previously [18-22]. A549 RIG-I OE cells were generated by stable lentiviral transduction as described previously [19]. Cells transduced with non-targeting gRNA (sequence taken from the GeCKO CRISPR v2 library) were used as controls. PH5CH non-neoplastic hepatocytes and HepG2 cells were kindly provided by Dr. Volker Lohmann (Heidelberg University, Heidelberg, Germany). Huh7.5 cells were generously provided by Dr. Charles Rice (Rockefeller University, New York).

Stimulation was performed with doxorubicin (DOX, Hölzel Diagnostika, Cologne, Germany), etoposide (ETO, Cell Signaling Technology, Danvers, MA, USA), or cells were transfected with in vitro transcribed and chromatographically purified $200 \mathrm{bp}$ 5'ppp-dsRNA [23], poly(C) (SigmaAldrich), and poly(I:C) (Sigma-Aldrich) using Lipofectamine 2000 (Invitrogen, Carlsbad, CA, USA) following the manufacturer's protocol. Cells were $\gamma$-irradiated with doses of 0-30 Gy using a Gammacell 40 Exactor (Best Theratronics, Ottawa, Canada).

Real-time imaging of cell death. A549 cells stably expressing histone H2B mCherry [21] were seeded at density of $2 \times 10^{3}$ cells per 96-well. The next day, cells were stimulated with 1-2 $\mu \mathrm{M}$ DOX $(10 \mathrm{~h})$, $25 \mu \mathrm{M}$ ETO (10 h), $0.1 \mathrm{ng} / \mathrm{ml}$ dsRNA ( 8 h), or $\gamma$-IR. DMSO (Carl Roth, Karlsruhe, Germany), poly(C), and mock irradiation were used as appropriate controls. Post treatment, fresh medium was supplemented with 1:10 000 IncuCyte ${ }^{\circledR}$ Cytotox Green Reagent (Sartorius, Göttingen, Germany) to determine dead cells. Total cell number and dead cells were monitored every $2 \mathrm{~h}$ using a $10 \mathrm{x}$ magnification in an IncuCyte ${ }^{\circledR}$ S3 Live-Cell Analysis System (Satorius, Göttingen, Germany). For IFN pre-stimulation, $200 \mathrm{IU} / \mathrm{ml}$ IFN- $\beta$ (IFN- $\beta 1$, Bioferon, Laupheim, Germany) or IFN- $\gamma$ (R\&D Systems, Minneapolis, MN, USA) were added at the time of seeding. For inhibitor administration, $40 \mu \mathrm{M} \mathrm{Z}$ VAD-FMK (Z-VAD, R\&D Systems) and $10 \mu \mathrm{M}$ Necrostatin-7 (Nec-7, Sigma Aldrich), or $25 \mu \mathrm{M}$ TPCA-1 (Sigma Aldrich) were added $2 \mathrm{~h}$ prior treatment. IncuCyte ${ }^{\circledR}$ Software (2019B Rev2, Satorius, Göttingen, Germany) was used to mask cells in phase contrast images. Calculations were performed applying the following settings: red fluorescence: segmentation top-hat, radius $100 \mu \mathrm{M}$, threshold (GCU) 0.4, edge split sensitivity -35 , area $60-1000 \mu \mathrm{m}^{2}$, integrated intensity $\geq 60$; green fluorescence: segmentation top-hat, radius $100 \mu \mathrm{M}$, threshold (GCU) 10, edge split sensitivity -40, area 100-700 $\mu \mathrm{m}^{2}$, eccentricity $\leq 0.8$, mean intensity 7-1000, integrated intensity $\geq 2500$. Percentage of dead cells was calculated relative to total cell count. Data represent the results of at least three biologically independent experiments. For curve charts, results were normalized to the control cell line of each replicate. Bars represent non-normalized means $36 \mathrm{~h}$ post treatment.

Immunofluorescence microscopy and determination of cellular IRF3 distribution. Fluorescence microscopy was performed to visualize phosphorylated histone H2A.X. After $4 \mathrm{~h}$ treatment with $2 \mu \mathrm{M}$ DOX or DMSO, or $1 \mathrm{~h}$ post $\gamma$-IR with $20 \mathrm{~Gy}$ or $0 \mathrm{~Gy}$, cells were permeabilized with $-20^{\circ} \mathrm{C}$ methanol and fixed with $4 \%$ paraformaldehyde. To block non-specific background, cells were incubated with $1 \%(\mathrm{w} / \mathrm{v})$ bovine serum albumin (BSA) and $10 \%(\mathrm{v} / \mathrm{v})$ FCS for $30 \mathrm{~min}$. Primary antibodies specific for phospho-H2A.X (Cell Signaling Technology, 9718, 1:1000) were applied at $4{ }^{\circ} \mathrm{C}$ over-night. Slides 
were incubated with Alexa Fluor ${ }^{\circledR} 488$ anti-rabbit (ThermoFisher Scientific, Waltham, MA, USA, A11008, 1:1000) and DAPI (ThermoFisher Scientific, D1306, 1:5000) for $1 \mathrm{~h}$. For determination of cellular IRF3 distribution, A549 cells stably expressing IRF3-eGFP and histone H2B-mCherry were stimulated either with DOX or poly(I:C) for $12 \mathrm{~h}$. Fluorescence was visualized using a Primovert microscope (Carl Zeiss, Jena, Germany).

Immunoblotting. Stimulated cells were lysed in Laemmli sample buffer, and digested with Benzonase ${ }^{\circledR}$ Nuclease (Merck Millipore, Burlington, MA, USA). For inhibitor administration, $20 \mu \mathrm{M}$ KU-55933 (Sigma-Aldrich), $25 \mu \mathrm{M}$ Rabusertib (Hölzel Diagnostika), $25 \mu \mathrm{M}$ TPCA-1 (Sigma Aldrich), or $10 \mu \mathrm{M}$ VE-822 (Hölzel Diagnostika) were added $2 \mathrm{~h}$ prior treatment. For stimulation with IFNs, $200 \mathrm{IU} / \mathrm{ml}$ IFN- $\alpha$ (PBL Assay Science, Piscataway, NJ, USA), IFN- $\beta$, or IFN- $\gamma$ were applied over-night. Lysed samples were further denatured at $95^{\circ} \mathrm{C}$ for $5 \mathrm{~min}$ and cleared from detritus. Resulting protein extracts were subjected to $10 \%(\mathrm{w} / \mathrm{v})$ SDS-polyacrylamide gel electrophoresis and transferred to PVDF membranes (Bio-Rad, Hercules, CA, USA, $0.2 \mu \mathrm{m}$ pore size). Upon incubation with $5 \%$ (w/v) BSA for $2 \mathrm{~h}$ to block non-specific background, membranes were probed using antibodies specific for $\beta$-actin (Sigma-Aldrich, A5441, 1:5000), calnexin (Enzo Biochem, Farmingdale, NY, USA, ADI-SPA-865-F, 1:1000), CASP3 (Cell Signaling Technology, 9662S, 1:1000), CASP9 (Cell Signaling Technology, 9508, 1:1000), IRF1 (Cell Signaling Technology, 8478S, 1:1000), phospho-IRF3 (pS396, ThermoFisher Scientific, MA5-14947, 1:1000), JAK1 (Cell Signaling Technology, 3332S, 1:1000), MDA5 (Enzo Biochem, ALX-210-935, 1:1000), NFKB1 (p50) (Abcam, Cambridge, UK, ab32360, 1:1000), p53 (Santa Cruz Biotechnology, Dallas, TX, USA, sc-126, 1:1000), or STAT1 (BD Biosciences, Franklin Lakes, NJ, USA, 610115, 1:1000) at $4{ }^{\circ} \mathrm{C}$ over-night. For detection, anti-rabbit horseradish peroxidase (HRP) (Sigma-Aldrich, A6154-5X1ML, 1:20 000) or anti-mouse HRP (SigmaAldrich, A4416-5X1ML, 1:10 000) were applied for $1 \mathrm{~h}$, membranes were covered with Amersham ECL Prime Western Blotting Detection Reagent (ThermoFisher Scientific) for $1 \mathrm{~min}$, and luminescence was detected using a sensitive CCD camera system (ECL ChemoCam Imager 3.2, INTAS Science Imaging Instruments, Göttingen, Germany). Densitometric analysis of the protein bands was performed using ImageJ (1.52e). Data shown represent the results of at least three biologically independent experiments.

Quantitative PCR with reverse transcription (qRT-PCR). Upon stimulation, cells were lysed and total RNA was isolated with the Monarch RNA isolation kit (New England Biolabs, Ipswich, MA, USA), following the manufacturer's protocol. After extraction, complementary DNA (cDNA) was generated using the High Capacity cDNA Reverse Transcription kit (ThermoFisher Scientific). Determination of messenger RNA (mRNA) expression was performed using iTaq Universal SYBR ${ }^{\circledR}$ Green Supermix (Bio-Rad) on a CFX96 real-time-system (Bio-Rad). Sequences of specific exonspanning PCR primers are shown in Supplementary Table S2. GAPDH mRNA was used as a housekeeping gene control and relative expression determined by $2^{\Delta \mathrm{Ct}}$ (thus, not normalizing to reference condition). 
161 Cell Viability. A549 cells were seeded at a density of $6 \times 10^{3}$ cells per 96-well. Upon treatment with $1622 \mu \mathrm{M}$ DOX or DMSO for $24 \mathrm{~h}$, cell viability was determined using the CellTiter-Glo ${ }^{\circledR}$ luminescent cell viability assay (Promega, Madison, WI, USA) following the manufacturer's protocol. Luciferase activity was measured using a Mithras LB 943 multimode reader (Berthold Technologies, Bad Wildbad, Germany).

166 Caspase activity. A549 cells were seeded at density of $6 \times 10^{3}$ cells per 96 -well. $48 \mathrm{~h}$ post treatment with $0-2 \mu \mathrm{M}$ DOX for $10 \mathrm{~h}$, caspase-3/7 activity was determined using the Apo-ONE ${ }^{\circledR}$ homogeneous caspase-3/7 assay (Promega) following the manufacturer's instructions. Resulting fluorescence was measured using the Mithras LB 943 multimode reader (Berthold Technologies).

\section{Statistics}

171 Comparison of datasets was performed using a paired, two-tailed Student's t-test. * indicates $\mathrm{p} \leq 0.05$, $* * \mathrm{p} \leq 0.01, * * * \mathrm{p} \leq 0.001, * * * * \mathrm{p} \leq 0.0001$. Error bars represent standard deviation.

\section{$\underline{\text { Results }}$}

\section{Apoptosis induction via DNA damage response pathway in A549 cells}

To investigate the molecular links between DNA damage-induced cell death and innate immune signaling, we used immunocompetent A549 human lung carcinoma cell lines with functional knockouts (KOs) of components of both pathways. Cells were treated with DNA DSB inducers, specifically $\gamma$-IR or the topoisomerase II inhibitors doxorubicin (DOX) and etoposide (ETO), and the resulting cell death was monitored on single-cell level by real-time imaging. Treatment of A549 cells with DOX resulted in pronounced cell death (Fig. 1A) and a corresponding reduction of bulk cell viability (Fig. 1B), accompanied by the detection of the DNA damage marker phospho-histone H2A.X by immunofluorescence (Fig. 1C). As in DMSO control conditions no cell death was observed (Fig. 1A), for the clarity of presentation we omitted this control in the following figures (data was acquired in every experiment). In order to characterize the type of cell death predominant upon DOX-induced DNA damage, we first evaluated activation of caspase- 3 and -7 being pivotal markers of apoptosis. DOX treatment activated caspase-3 and -7 in a dose-dependent manner (Fig. 1D). Conversely, we treated cells with the pan-caspase inhibitor Z-VAD, or depleted caspase-3 or -9. Both approaches resulted in a significant reduction of cell death upon DOX treatment (Fig. 1E, F, $\mathrm{H})$. These findings confirmed prior reports that cell death driven by DOX is mainly due to apoptosis [24]. Next, we investigated typical components of the DNA damage response upstream of caspase activation. In line with p53's (TP53) essential role in inducing apoptosis, depletion of p53 showed a significant reduction of cell death (Fig. $1 \mathrm{G}, \mathrm{H}$ ). Interestingly, TP53 ${ }^{-/-}$had the opposite effects at late time points, elevating cell death for time points $>54 \mathrm{~h}$ (Fig. 1G). Amongst others, p53 induces apoptosis via activation of PUMA and NOXA. Accordingly, we found PUMA and NOXA transcript levels to be 
A
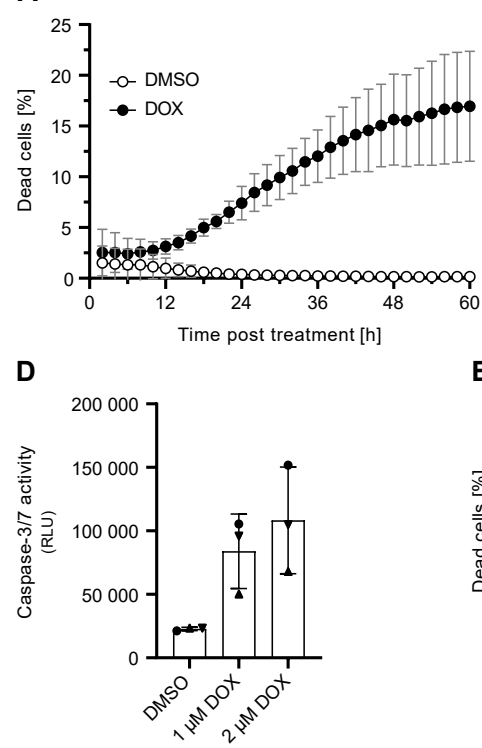

G

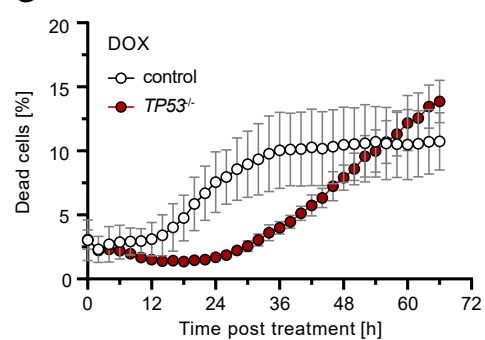

B

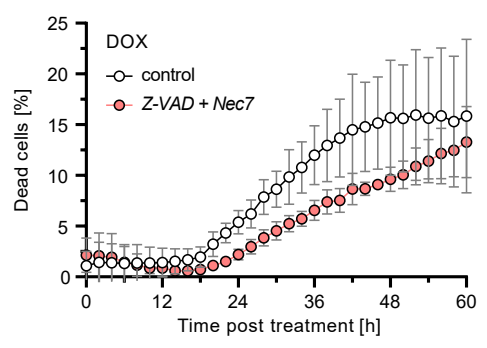

H

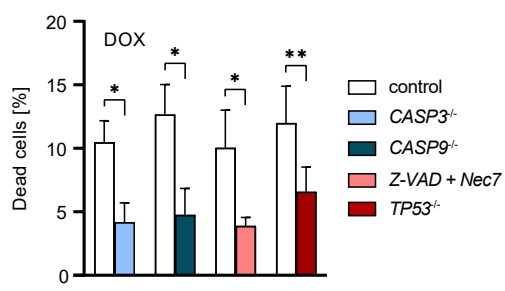

C
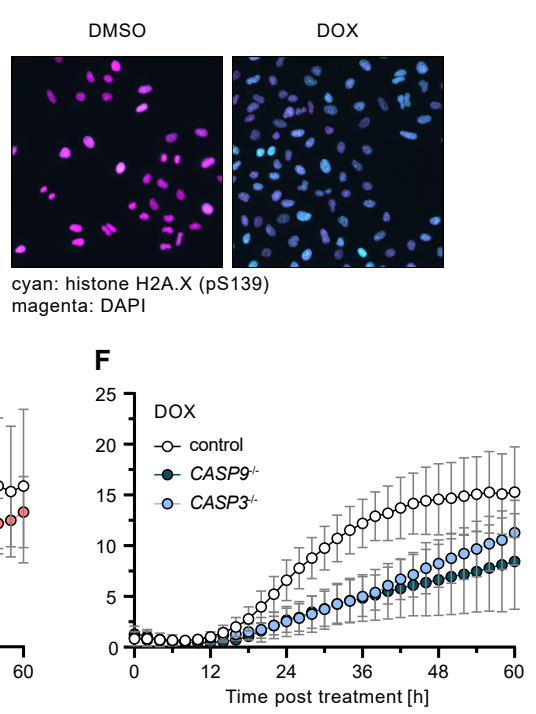

I

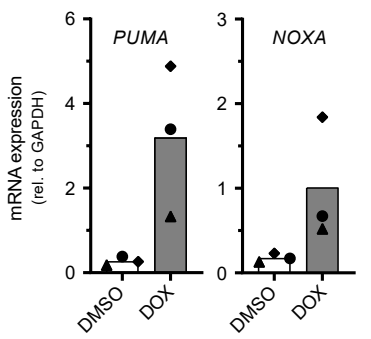

Fig. 1. Induction of apoptosis upon DOX-mediated DNA damage.

(A) Percentage of dead A549 cells relative to total cells counted over time post DOX or DMSO treatment. (B) Cell viability of A549 cells post DOX treatment for $24 \mathrm{~h}$. (C) Immunofluorescence of phosphorylated histone H2A.X (S139) (cyan) and DAPI-stained nuclei (magenta) in A549 cells post DOX treatment for 4 h. (D) Caspase3/7 activity of A549 cells 24 h post DOX treatment for 10 h. (E-H) Percentage of dead A549 cells with caspase inhibition or functional KO of the indicated genes relative to total cells counted over time (E-G) or $36 \mathrm{~h}(\mathbf{H})$ post DOX treatment. (I) A549 cells were treated with $1 \mu \mathrm{M}$ DOX or DMSO for $24 \mathrm{~h}$. PUMA and NOXA mRNA transcripts were determined by qRT-PCR. (A, B, D-I) Data shown represent the results of at least three biologically independent experiments.

\section{Relevance of innate antiviral immunity pathways in DNA damage induced cell death}

In order to investigate the contribution of antiviral signaling cascades to the induction of DSB-induced cell death, we compared the impact of the major antiviral pathways using KOs of their respective signaling adapters. We observed DOX-induced cell death to be significantly reduced only by MAVS depletion (RLR signaling), but not so in the absence of STING (cGAS signaling), TRIF (TLR3 signaling), or MYD88 (general TLR signaling) (Fig. 2A-C). Despite RLR signaling appeared to play a major role, neither canonical IRF3 phosphorylation nor its nuclear translocation could be detected 
215 (Fig. 2D, E). Consistently, there was also no characteristic RLR-mediated induction of ISGs, such as $216 \operatorname{IFIT1}$ (Fig. 2F).

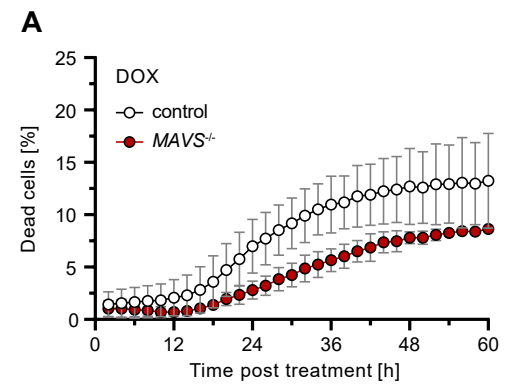

D

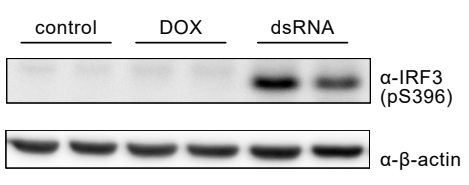

\section{B}

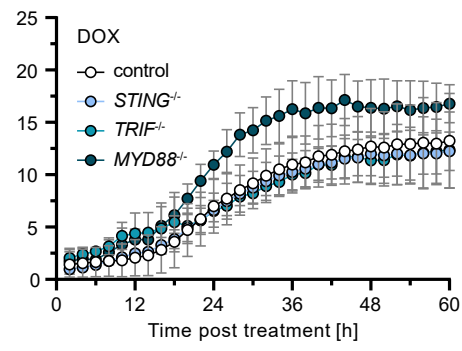

E

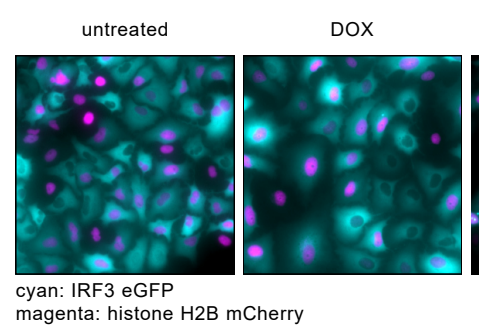

C

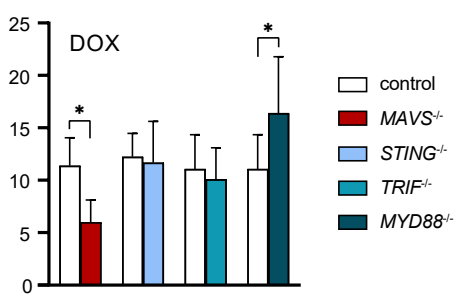

F

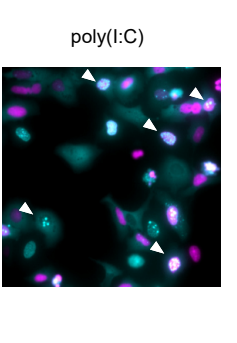

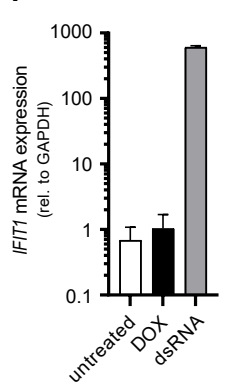

217

218

219

220

221

222

223

224

225

226

227

228

229

230

231

232

233

234

235

236

237

238

Fig. 2. Relevance of antiviral signaling adapters and ISG response during DOX-induced DNA damage response.

(A-C) Percentage of dead A549 cells with functional KO of the indicated genes relative to total cells counted over time (A, B) or $36 \mathrm{~h}$ (C) post DOX treatment. (D) A549 cells were stimulated with $1 \mu \mathrm{M}$ DOX or $1 \mathrm{ng} / \mathrm{ml}$ dsRNA for 8 h. Phosphorylated IRF3 (S396) was determined by western blot. (E) A549 cells were stimulated with $1 \mu \mathrm{M}$ DOX or $2 \mu \mathrm{g} / \mathrm{ml}$ poly(I:C) for $12 \mathrm{~h}$. Cellular distribution of IRF3 eGFP (cyan) and histone H2B (magenta) was visualized by immunofluorescence microscopy. (F) A549 cells were stimulated with $1 \mu \mathrm{M}$ DOX or $10 \mathrm{ng} / \mathrm{ml}$ dsRNA for $24 \mathrm{~h}$. IFIT1 mRNA transcripts were determined by qRT-PCR. (A-C, F) Data shown represent the results of at least three biologically independent experiments.

Given the observed relevance of MAVS in DOX-induced cell death, we further analysed the effect of specific RLR depletion. Both $R I G-I^{-/}$and $M D A 5^{-/-}$reduced cell death upon DOX treatment, however, RIG-I exhibited a considerably stronger effect (Fig. 3A, C). Reciprocally, RIG-I overexpression (OE) markedly increased cell death upon DOX treatment (but not in untreated conditions, compare Fig. S1A), underlining the decisive role of RLR signaling in this process (Fig. 3B, C). In order to determine the factors responsible for mediating cell death downstream of MAVS, we further examined the influence of transcription factors IRF3 and NF-kB p65/RELA. We observed that depletion of either factor significantly reduced DOX-induced cell death (Figure 3D, F). Using IFN-“blind” A549 IFNAR ${ }^{-/}$ $I F N L R 1^{-/-} I F N G R^{-/-}$(IFNR TKO) cells, we demonstrated that this effect was independent of a response mediated by secreted IFNs (Fig. 3E, F), which was further confirmed using $S T A T 1^{-/}$cells (Fig. S1B). This was in accordance with the lack of ISG expression observed previously (Fig. 2F). Thus, IRF3 
appears to have death sensitizing effects distinct from its classical transcriptional activity in the antiviral program.

241 Taken together, we demonstrated that RLR signaling is required for the induction of cell death after 242 DNA damage and that this function is independent of IFN secretion and the induction of canonical 243 ISGs.

A

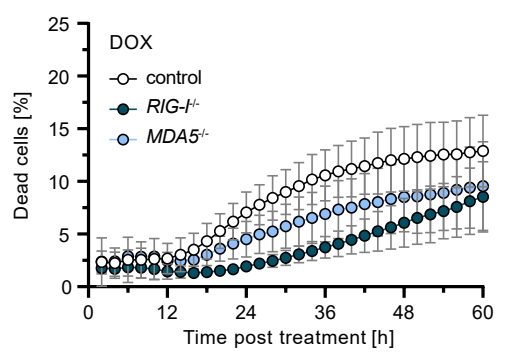

D

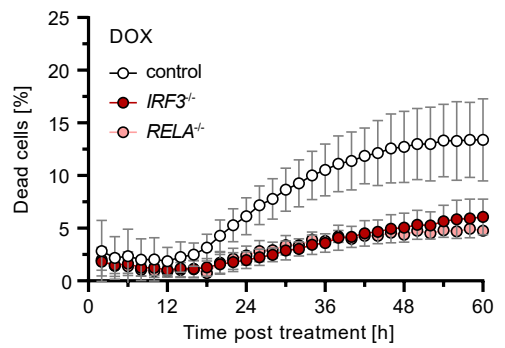

B

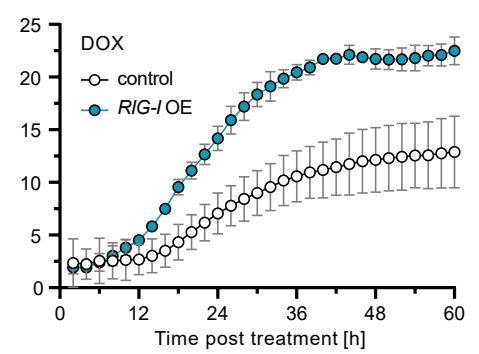

E

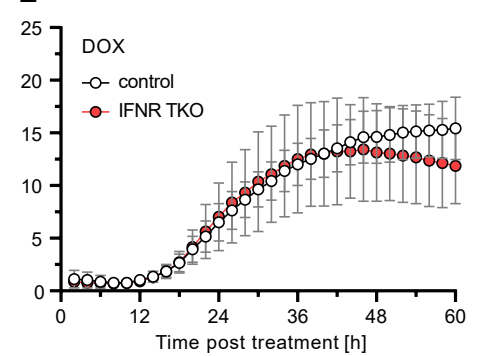

C

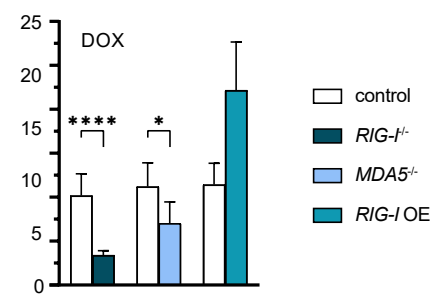

$\mathbf{F}$

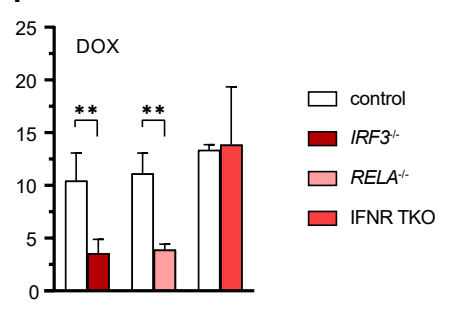

Fig. 3. Implications of RLR signaling components and IFN signaling on DOX-induced apoptosis.

(A-F) Percentage of dead A549 cells with functional $\mathrm{KO}$ or OE of the indicated genes relative to total cells counted over time (A, B, D, E) or $36 \mathrm{~h}(\mathbf{C}, \mathbf{F})$ post DOX treatment. Data shown represent the results of at least three biologically independent experiments.

\section{Role of IRF1 in DNA damage induced apoptosis}

Another transcription factor of the IRF family important for antiviral defenses [6, 18], IRF1, has previously also been implicated with the DNA damage response [25]. We hypothesized that upon genotoxic insult, IRF1 might be a downstream target of the RLR/IRF3 pathway, as reported for virus infection, and thereby link RLR activity to the DNA damage response. Indeed, upon DOX treatment, we observed IRF1 upregulation at the mRNA (Fig. 4A) and protein level (Fig. 4B). Of note, IRF1 induction occurred independently of the presence of p53 (Fig. 4B). In order to determine the relevance of IRF1 to cell death, we next tested $I R F 1^{-/}$cells in DOX treatment. Strikingly, IRF1 depletion almost completely abolished DOX-induced cell death (Fig. 4E, H). Conversely, increasing IRF1 abundance, either by OE through stable transduction or by pre-stimulation of cells with IFN- $\beta$ or IFN- $\gamma$, markedly increased cell death upon DOX treatment (Fig. 4E, F, H), and the percentage of dead cells correlated with IRF1 levels in western blot (Fig. 4C, D). Notably, neither IFN stimulation alone, nor DOX treatment in IFN-primed but IRF1-depleted cells did induce cell death (Fig. S2A, B). Surprisingly, the same phenotype was observed in $R I G-I^{-/}$conditions (Fig. S2C), in which IRF1 was present, suggesting 
a strict requirement of both RLR signaling and $I R F 1$ induction for proper triggering and/or execution of apoptosis. Similar observations were also made after ETO treatment (Fig. S2D, E), ruling out DOXspecific effects.

267 The fundamental importance of IRF1 was additionally demonstrated in response to $\gamma$-IR. Although irradiation did induce DNA damage in A549 cells (Fig. S2F), we could neither observe induction of IRF1 expression nor any cell death upon administration of up to $30 \mathrm{~Gy}$ (Fig. 4G-I). Strikingly, induction of cell death upon $\gamma$-IR was restored under conditions of elevated IRF1 levels, such as stable OE or IFN- $\gamma$ pre-stimulation (Fig. $4 \mathrm{G}, \mathrm{H}$ ). In line with this, cells in which $\gamma$-IR naturally leads to an upregulation of IRF1 expression, such as $\mathrm{PH} 5 \mathrm{CH}$ cells, did exhibit a dose-dependent induction of cell death (Fig. S2G, H). death upon DNA damage. IFNs, in particular IFN- $\gamma$, sensitize cells for DNA damage-induced apoptosis through upregulation of IRF1.

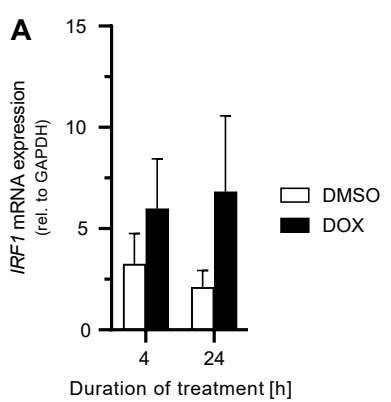

E
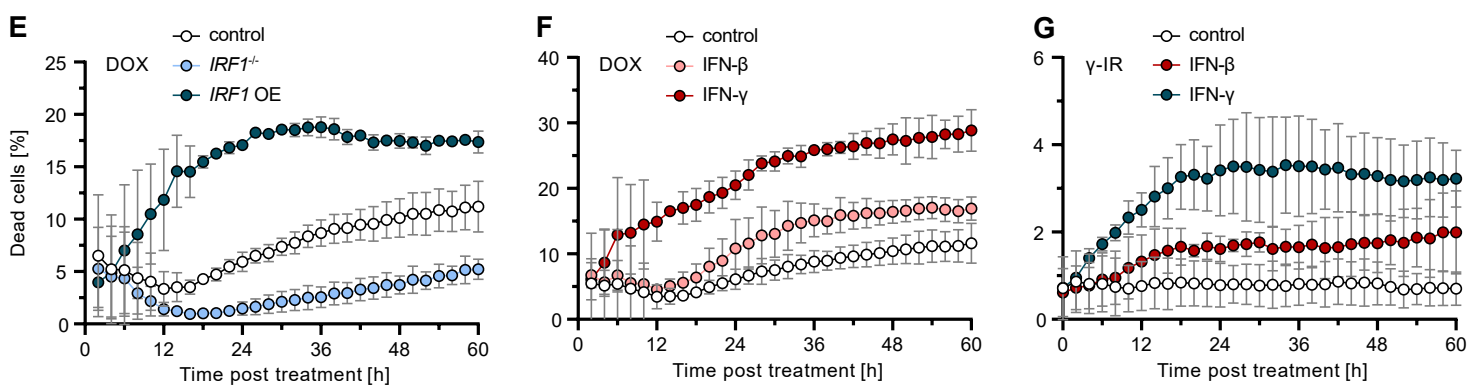

H
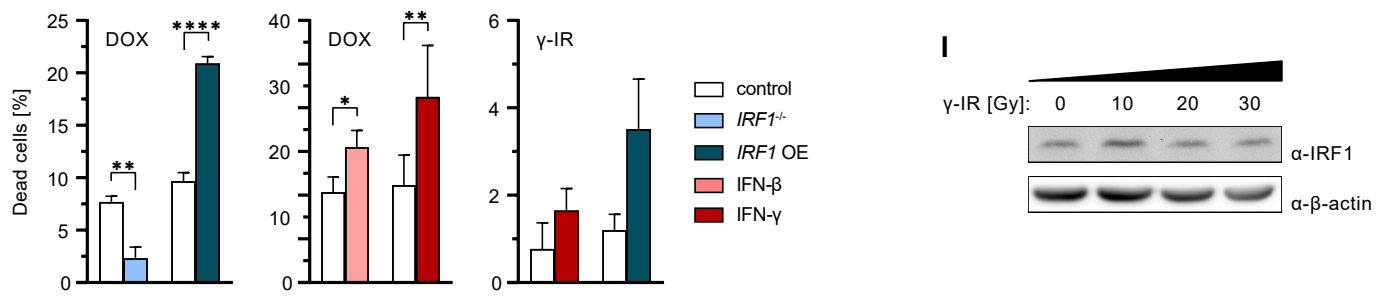

B
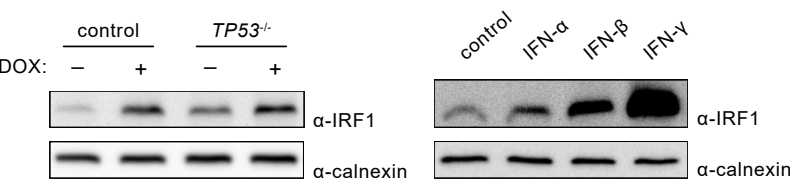

\section{Fig. 4. Relevance of IRF1 on DNA damage-induced cell death.}

(A) A549 cells were treated with $1 \mu \mathrm{M}$ DOX or DMSO for $10 \mathrm{~h}$. IRF1 mRNA transcripts were determined by qRT-PCR. (B) A549 cells were treated with $1 \mu \mathrm{M}$ DOX or DMSO for $10 \mathrm{~h}$. Levels of IRF1 were determined by western blot. (C) A549 cells were stimulated with IFN- $\alpha$, IFN- $\beta$, or IFN- $\gamma$ over-night. Levels of IRF1 were determined by western blot. (D) Levels of IRF1 in A549 control and IRF1 OE cells were determined by western 
blot. (E-H) Percentage of dead A549 cells with functional KO or OE of IRF1, or post IFN pre-stimulation relative to total cells counted over time (E-G) or $36 \mathrm{~h}(\mathbf{H})$ post DOX or $\gamma$-IR (20 Gy) treatment. (I) A549 cells were $\gamma$ irradiated. After $10 \mathrm{~h}$ IRF1 protein levels were determined by western blot. (A, E-H) Data shown represent the results of at least three biologically independent experiments.

\section{Regulation of IRFI expression upon DNA damage}

Above we have shown that RLR/IRF3 signaling as well as expression of IRF1 are crucially important for DNA damage-induced cell death. We further found IRF1 to be consistently induced under all tested conditions of DNA damage leading to cell death. We now aimed to confirm whether IRF1 is in fact induced as a downstream target of RLR signaling. We first investigated the induction of IRF1 expression after RIG-I stimulation using dsRNA as a canonical, highly specific agonist [23]. Indeed, we observed a fully RLR-dependent (RIG-I, MAVS, IRF3) increase of IRF1 levels, with a partial contribution of p65/RELA and IFN signaling (IFNR TKO) (Fig. 5A), in line with a recent report of our lab [18]. dsRNA-stimulation furthermore also led to the induction of cell death, which was fully abolished upon depletion of the RLR signaling components RIG-I, MAVS, or IRF3 (Fig. 5B, D). Depletion of p65/RELA and the IFN receptors (IFNR TKO) had minor pro-survival effects, suggesting a major role for transcription-independent RIPA with a possible but limited role for IFN signaling and ISG induction (Fig. 5C, D). Interestingly and in clear contrast to the situation upon DNA damage, dsRNA-induced cell death was independent of IRF1 (Fig. 5C). Nonetheless, experimentally elevating IRF1 levels markedly increased the percentage of dead cells also in this setting (Fig. S3A, B).

These findings confirmed that, despite not being essential for cell death induction, IRF1 is induced downstream of RLR signaling, at least when stimulated by a strong RIG-I specific agonist. We next investigated whether this would be also the case in the context of DNA damage. Unexpectedly, upon treatment of cells with DOX, induction of IRFI expression was neither affected by depletion of RLR nor of IFN signaling components, including JAK1 (Fig. 5E, F; Fig. S3C). This suggested IRF1 expression is induced independently of and coincidentally with antiviral RLR signaling upon DNA damage. We therefore hypothesized sensing of DNA damage might directly induce $I R F 1$. To test this, we treated cells with specific inhibitors of the prototypical DSB sensors ATM and ATR, as well as potential downstream pathways. We found IRFI induction upon DOX-treatment to be completely blocked by the ATM inhibitor KU-55933 [26] and the ATR inhibitor VE-822 [27], suggesting important roles of these sensors in activation of IRF1 (Fig. 5G; Fig. S3D).

As IRF1 expression has previously been shown to be NF-kB sensitive [28], we employed the common pan-NF-kB and JAK1 inhibitor TPCA-1 [29, 30]. Remarkably, TPCA-1 treatment completely prevented the induction of IRFI expression upon DOX treatment, and even strongly diminished basal expression (Fig. 5G, Fig. S3D). This effect could further be confirmed upon RLR-stimulation with dsRNA (Fig. S4A) and even upon IFN- $\gamma$ treatment, which is a strong and well-studied canonical inducer of IRF1 (Fig. S4B). We could rule out a cell line (A549) specific effect by testing three other human 
bioRxiv preprint doi: https://doi.org/10.1101/2021.10.21.465312; this version posted October 22, 2021. The copyright holder for this preprint (which was not certified by peer review) is the author/funder, who has granted bioRxiv a license to display the preprint in perpetuity. It is made available under aCC-BY-NC-ND 4.0 International license.

cell lines, PH5CH, HeLa and Huh7.5 (Fig. S4C). To our knowledge, this striking effect of TPCA-1 on IRF1 expression has not been reported before. Again, corroborating IRF1's crucial role in DNA damage-induced apoptosis, supressing IRFI induction by TPCA-1 also reduced cell death in DOXtreated A549, PH5CH, HeLa, and Huh7.5 cells (Fig. S4D).

Finally, we aimed to identify which signaling pathway and NF- $\mathrm{B}$ subunit would be responsible for $I R F 1$ expression upon triggering the DNA damage response. As reported in literature, ATR may signal through CHK1 to activate p50/NFKB1, a potential target of TPCA-1 [31, 32]. We therefore inhibited CHK1 by Rabusertib [33] prior to DOX-treatment. However, our experiments did not reveal any effect of CHK1 inhibition or p50/NFKB1 depletion on IRF1 levels (Fig. 5G; Fig. S3D, E). We hence conclude that a so far elusive pathway downstream of the ATM/ATR system induces IRFI.

Taken together, we demonstrated that $I R F 1$ expression upon DOX-treatment is induced by the DSB sensors ATM/ATR rather than RLR signaling. This induction is independent of CHK1 signaling. Additionally, we identified a previously unappreciated IRF1-depleting effect of the NF- $\mathrm{KB}$ inhibitor TPCA-1.

A

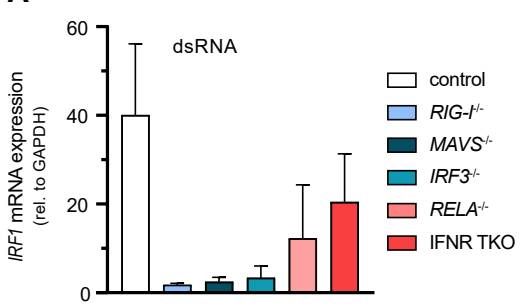

D

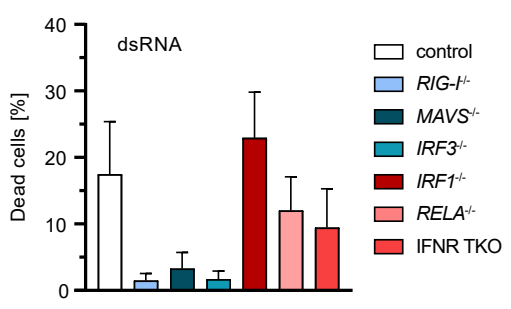

B
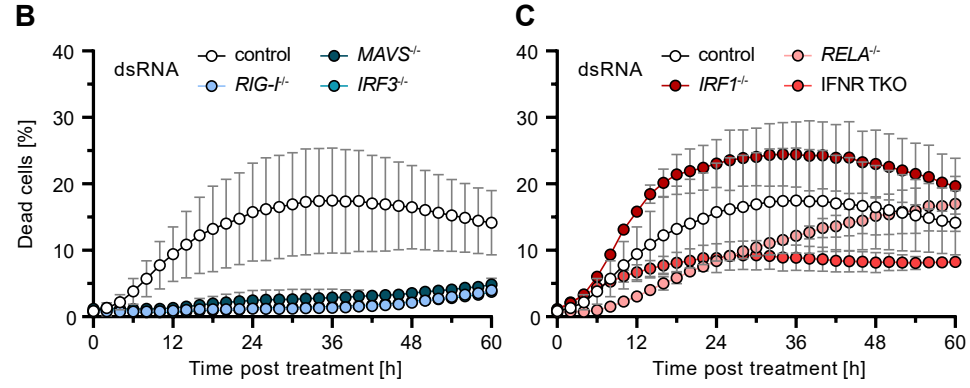

E

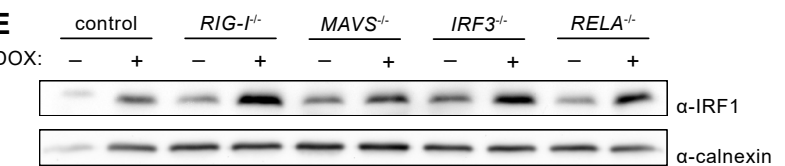

FOX: $\frac{\text { control }}{-+} \frac{\text { JAK1\% }}{-} \frac{\text { IFNR TKO }}{-++}$

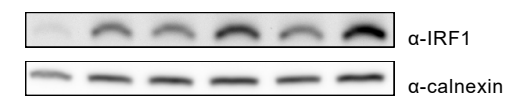

G
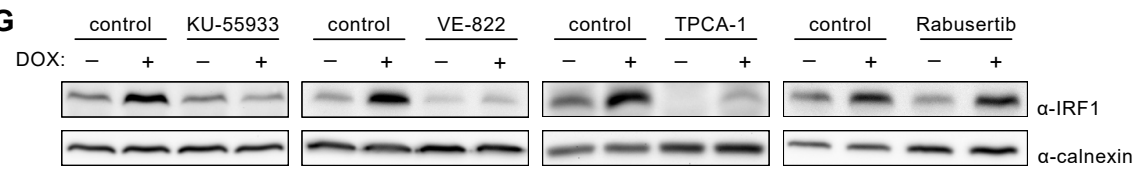

Fig. 5. Effect of cell-intrinsic antiviral signaling components on dsRNA-induced cell death and IRFI expression.

(A) A549 cells with functional $\mathrm{KO}$ of the indicated genes were stimulated with $2 \mathrm{ng} / \mathrm{ml}$ dsRNA for $6 \mathrm{~h}$. IRF1 mRNA transcripts were determined by qRT-PCR. (B-D) Percentage of dead A549 cells with functional KO of the indicated genes relative to total cells counted over time (B, C) or $36 \mathrm{~h}$ (D) post dsRNA stimulation. (E-G) A549 cells with functional $\mathrm{KO}$ of the indicated genes or administration of the indicated inhibitors were treated with 
$2 \mu \mathrm{M}$ DOX or DMSO for $6 \mathrm{~h}$. Levels of IRF1 were determined by western blot. (A-D) Data shown represent the results of at least three biologically independent experiments.

\section{$\underline{\text { Discussion }}$}

Cells, particularly of multicellular organisms, have elaborate systems in place ensuring the integrity of their genome, as DNA damage poses severe risks of accumulating tumorigenic mutations or alterations. In response to excessive DNA damage beyond the potential of being properly repaired, cells trigger the execution of cell death programs, most commonly apoptosis [34]. This is also exploited for common cancer chemoradiotherapies, in which excessive DNA damage is radiologically (e.g., $\gamma$-IR) or pharmacologically (e.g., DOX or ETO) introduced, leading to the induction of cell death programs particularly in dividing tissues such as tumors. Elucidating the underlying mechanisms of how DNA damage molecularly leads to cell death is crucial to a better understanding of the circumstances leading to cancer and the pathways relevant for chemoradiotherapy. While classical DNA damage checkpoint control via p53 has been investigated thoroughly [1], much less is known about the relevance and contribution of non-canonical pathways. For example, a ground-breaking study surprisingly found the antiviral type I IFN pathway essential for certain chemotherapies' efficacy [35]. Cytostatic and proapoptotic effects of IFNs have long been noticed [36-38]; however, it remained unresolved what triggered the production of IFNs in the studied context in the first place. Recent data also revealed cellintrinsic triggering of cell death upon activation of antiviral signaling adapters, such as MAVS and STING. Interestingly, this was not only the case for viral infections, but also in response to DNA damage $[10,11,39]$.

In the present study, we confirm this interrelationship between DNA damage response and antiviral signaling pathways, and we demonstrate an almost complete dependence of DOX-and ETO-triggered cell death on the presence of intact RLR/MAVS signaling. In clear contrast to recently published data, other branches of the cell-intrinsic antiviral defense, such as the TLR or the cGAS/STING system [10, 40, 41], did not affect DOX-induced cell death in our experimental setup. Instead, the cytosolic RNA sensors RIG-I and, to a lesser extent, MDA5 were triggered and essential for the induction of cell death. This is in line with a study by Ranoa et al. suggesting small nuclear RNAs U1 and U2 translocate into the cytoplasm in irradiated cells and trigger RIG-I activation [11]. In our experimental system, an intact RIG-I/MDA5-MAVS-IRF3 axis was essential for DNA damage induced cell death; however, we could not observe canonical transcriptional activities of IRF3, such as the induction of IFN genes or ISGs. While the relevance of both IRF3 and p65/RELA suggested the involvement of IFNB expression, KO of the receptors for all three types of IFNs (IFNR TKO) did not impact cell death. A plausible mechanism for this IFN-independent triggering of apoptosis is RIPA, involving LUBAC-dependent ubiquitylation of IRF3 and subsequent activation of pro-apoptotic BH3-only proteins [16]. The clear contribution of p65/RELA in our experiments might be through its transcriptional activation of further 
pro-apoptotic proteins [42]. To our knowledge, cooperative effects between RIPA and NF- $\kappa B$ have not been described before and may be an interesting subject for future investigations.

Efficient sensing of nuclear DSBs and triggering an appropriate response is critical for cell survival upon DNA damage, or for initiating cell death and preventing potentially cancerous transformation. As expected, we observed an essential role for p53, highlighting its central function in checkpoint control, coordinating DNA damage repair and triggering apoptosis as a last resort [43]. Interestingly, depletion of 553 reduced the number of apoptotic cells at early time points, but increased cell death at later times. Thus, absence of $\mathrm{p} 53$ led to a lack of induction of apoptosis in response to DOX-mediated DSBs at first, but likely massive accumulation of unrepaired DNA damage eventually led to increased, putatively necrotic cell death [44]. As a factor potentially linking the DNA damage response and antiviral signaling, we investigated the role of the multifunctional transcription factor IRF1, as it is known to be involved in both the DNA damage response $[8,25]$ and IFN signaling $[6,18,45]$. Indeed, we found that IRF1 was considerably upregulated upon DOX and ETO treatment as well as $\gamma$-IR in different cell lines. Interestingly, only in A549 cells, described to be relatively radioresistant as a common characteristic for non-small cellular lung cancers [46], IRF1 was not appreciably induced upon irradiation. We also observed a reduced histone H2A.X phosphorylation after $\gamma$-IR compared to DOX treatment, but potential underlying mechanisms are only partially understood and may comprise several processes [47, 48]. Nonetheless, we could further corroborate this clear correlation between IRFI induction and triggering/execution of a cell death program on a functional level. Experimentally increasing IRF1 levels by stable OE or by pre-treatment of cells with IFN- $\gamma$, known as a strong inducer of IRF1 [45], radioresistance of A549 cells could be overcome. A similar effect has previously been demonstrated in $\mathrm{T}$ cells [25]. In our experiments, increased $I R F I$ expression also led to a sensitization towards DOXtreatment. Vice versa, IRF1 KO almost completely rescued cell survival upon DOX-, ETO- and $\gamma$-IRinduced DNA damage. These observations clearly establish a fundamentally important role of IRF1 in DNA damage-induced cell death. This is in accordance with literature suggesting IRF1 as a biomarker for radioresistance in tumor cells [49]. For example, extremely radioresistant osteosarcomas were shown to exhibit significantly reduced IRFI expression levels [50]. Our data further support establishing IRF1 as a predictive biomarker in chemoradiotherapy in tumor patients.

Our finding strongly suggested IRF1 to be the functional link between the DNA damage response and the antiviral system, with RLR signaling (either directly or via the IFN/JAK/STAT cascade) leading to transcriptional activation of IRF1. However, KO experiments clearly refuted this hypothesis. Neither $\mathrm{KO}$ of essential factors of the RLR pathway nor of IFN signaling components abolished IRFI induction upon DNA damage, suggesting that RLR signaling may activate IRF1 post-translationally. Generally, IRF1 is thought to be only regulated on a transcriptional level [45]. However, one study reports the requirement for "licensing" of IRF1 to become fully active, which required TLR signaling and MYD88 [51]. In preliminary experiments, we did not find any evidence for post-translational modifications in our setting, but this may warrant deeper investigations in the future. Alternatively, IRF1 might enhance 
the transcriptional response of IRF3, as reported before [52]. While we cannot rule out this possibility, the virtually complete inhibition of cell death in $I R F 1^{-/-}$despite abundant presence of IRF3 makes this unlikely. In another study, we have also not found any indication of a dampening of IRF3 responses in A549 $\mathrm{IRF}^{-/ /}$cells [18]. Notably, despite IRF1 being critically important for cell death induction in our system, IRF1 (over-)expression alone did not suffice to elicit apoptosis. We therefore suspect RLR signaling and IRF1 activity to cooperate further downstream, putatively via the transcriptional activation of complementary pro-apoptotic factors.

It is interesting to note that cell death is also elicited upon RLR stimulation by dsRNA (the canonical way to trigger antiviral signaling). Also in this case, IRFI is induced, but strictly dependent on RIG-I and to a lesser extent dependent on IFN signaling. Surprisingly, however, depletion of IRF1 did not affect the cell death rate upon dsRNA stimulation, pointing towards transcription-independent mechanisms such as RIPA [15]. Still, KO of NF-אB (RELA) or the IFN receptors (IFNR TKO) affect cell death, suggesting some transcriptional regulation, which, however, was independent of IRF1. This may suggest that full-fledged RLR signaling upon dsRNA encounter induces a sufficiently broad transcriptional response, which (in contrast to the situation upon DNA damage) itself is capable of triggering apoptosis. Strikingly, even in dsRNA stimulation, ectopic OE of IRFI or pre-treatment of cells with IFN- $\gamma$ led to a notable increase in the number of dying cells, putatively by the same cooperative pro-apoptotic effects observed in the case of DNA damage. This observation of a general sensitization for cell death by IRF1 is in line with data showing that IRFI OE enhances apoptosis in breast or gastric cancer treatment [53-55]. It is further plausible to speculate that reported pro-apoptotic effects of type I IFN $[56,57]$ would also be mediated by upregulation of IRF1 through homodimeric STAT1 transcription factor complexes (GAF) inadvertently formed early upon IFNAR engagement [58]. This could mechanistically explain how IFN- $\alpha$ improved chemotherapy response and overall survival in a murine tumor model [35]. Thus, evidence further accumulates suggesting IRFl-inducing agents to be more broadly considered as adjuvants in tumor therapy. Two central questions remain: firstly, which pro-apoptotic factors are specifically induced by IRF1 upon DNA damage that so potently sensitize cells to committing suicide upon (slight) RLR triggering. To this end, we are currently investigating IRF1-dependent candidate genes induced upon DOXtreatment at a transcriptomic level. Secondly, how is IRFI induced upon DNA damage in the first place if not through classical STAT1:STAT1 activity. In our study, we found its transcriptional regulation to be fully independent of RLR signaling and p53 but completely reliant on DNA DSB sensing via ATM and ATR. Still, the downstream pathway leading to IRF1 expression remains elusive. While p65/RELA or p50/NFKB1 depletion did not affect IRF1 induction, it was completely abolished by TPCA-1, a commonly known inhibitor of NF- $\mathrm{KB}$. Interestingly, TPCA-1 considerably reduced baseline IRF1 
4501 appears to specifically and very efficiently inhibit the activity of an essential transcription factor for 451 IRF1.

452 In conclusion, our study highlights the critical relevance of the antiviral RLR system for the proper and timely induction of cell death upon DNA damage. We provide evidence for independent but cooperative involvement of p53, IRF1 and IRF3 activity upon detection of DNA DSBs by the ATM/ATR machinery. We show that elevating expression levels of $I R F 1$ lead to the sensitization towards cell death across different genotoxic insults, such as chemotherapeutics, $\gamma$-IR or cytosolic dsRNA (i.e. virus infection). These data corroborate a fundamental role for IRF1 and RLR signaling in DNA damagemediated cell death and suggest future exploration of IRFI inducers, such as IFN- $\gamma$, together with lowdose RIG-I agonists for their potential as highly efficacious adjuvants in chemoradiotherapy. Additionally, our findings support IRF1 as a biomarker predictive for chemo- and radio-sensitivity of tumors.

\section{$\underline{\text { Literature }}$}

1. Sullivan, K.D., et al., The p53 circuit board. Biochimica et Biophysica Acta (BBA) Reviews on Cancer, 2012. 1825(2): p. 229-244.

2. Shiloh, Y. and Y. Ziv, The ATM protein kinase: regulating the cellular response to genotoxic stress, and more. Nature Reviews Molecular Cell Biology, 2013. 14(4): p. 197-210.

3. Shao, L., et al., Deficiency of the DNA repair enzyme ATM in rheumatoid arthritis. Journal of Experimental Medicine, 2009. 206(6): p. 1435-1449.

6. Fujita, T., et al., Evidence for a nuclear factor(s), IRF-1, mediating induction and silencing properties to human IFN-beta gene regulatory elements. The EMBO Journal, 1988. 7(11): p. 3397-3405.

7. Yamane, D., et al., Basal expression of interferon regulatory factor 1 drives intrinsic hepatocyte resistance to multiple RNA viruses. Nature Microbiology, 2019. 4(7): p. 1096-1104.

8. Harada, H., et al., Anti-oncogenic and oncogenic potentials of interferon regulatory factors-1 and -2. Science, 1993. 259(5097): p. 971. 
9. Doherty, G.M., et al., Interferon regulatory factor expression in human breast cancer. Annals of surgery, 2001. 233(5): p. 623-629.

10. Härtlova, A., et al., DNA Damage Primes the Type I Interferon System via the Cytosolic DNA Sensor STING to Promote Anti-Microbial Innate Immunity. Immunity, 2015. 42(2): p. 332-343.

11. Ranoa, D.R.E., et al., Cancer therapies activate RIG-I-like receptor pathway through endogenous non-coding RNAs. Oncotarget, 2016. 7(18): p. 26496-26515.

12. Elion, D.L., et al., Therapeutically Active RIG-I Agonist Induces Immunogenic Tumor Cell Killing in Breast Cancers. Cancer Research, 2018. 78(21): p. 6183.

13. Besch, R., et al., Proapoptotic signaling induced by RIG-I and MDA-5 results in type I interferon-independent apoptosis in human melanoma cells. The Journal of Clinical Investigation, 2009. 119(8): p. 2399-2411.

14. Goubau, D., et al., Transcriptional re-programming of primary macrophages reveals distinct apoptotic and anti-tumoral functions of IRF-3 and IRF-7. European Journal of Immunology, 2009. 39(2): p. 527-540.

15. Chattopadhyay, S., et al., Viral apoptosis is induced by IRF-3-mediated activation of Bax. The EMBO Journal, 2010. 29(10): p. 1762-1773.

16. Chattopadhyay, S., et al., Ubiquitination of the Transcription Factor IRF-3 Activates RIPA, the Apoptotic Pathway that Protects Mice from Viral Pathogenesis. Immunity, 2016. 44(5): p. 1151-1161.

17. El Maadidi, S., et al., A Novel Mitochondrial MAVS/Caspase-8 Platform Links RNA Virus-Induced Innate Antiviral Signaling to Bax/Bak-Independent Apoptosis. The Journal of Immunology, 2014. 192(3): p. 1171.

18. Wüst, S., et al., Comparative Analysis of Six IRF Family Members in Alveolar Epithelial Cell-Intrinsic Antiviral Responses. Cells, 2021. 10(10): p. 2600.

19. Willemsen, J., et al., Phosphorylation-Dependent Feedback Inhibition of RIG-I by DAPK1 Identified by Kinome-wide siRNA Screening. Molecular Cell, 2017. 65(3): p. 403-415.e8.

20. Krischuns, T., et al., Phosphorylation of TRIM28 Enhances the Expression of IFN- $\beta$ and Proinflammatory Cytokines During HPAIV Infection of Human Lung Epithelial Cells. Front Immunol, 2018. 9: p. 2229.

21. Urban, C., et al., Persistent Innate Immune Stimulation Results in IRF3-Mediated but Caspase-Independent Cytostasis. Viruses, 2020. 12(6). 
22. Bender, S., et al., Activation of Type I and III Interferon Response by Mitochondrial and Peroxisomal MAVS and Inhibition by Hepatitis C Virus. PLOS Pathogens, 2015. 11(11): p. e1005264.

23. Binder, M., et al., Molecular mechanism of signal perception and integration by the innate immune sensor retinoic acid-inducible gene-I (RIG-I). The Journal of biological chemistry, 2011. 286(31): p. 27278-27287.

24. Wang, S., et al., Doxorubicin induces apoptosis in normal and tumor cells via distinctly different mechanisms. intermediacy of $H(2) O(2)$ - and p53-dependent pathways. J Biol Chem, 2004. 279(24): p. 25535-43.

25. Tamura, T., et al., An IRF-1-dependent pathway of DNA damage-induced apoptosis in mitogen-activated T lymphocytes. Nature, 1995. 376(6541): p. 596-599.

26. Hickson, I., et al., Identification and Characterization of a Novel and Specific Inhibitor of the Ataxia-Telangiectasia Mutated Kinase ATM. Cancer Research, 2004. 64(24): p. 9152.

27. Fokas, E., et al., Targeting ATR in vivo using the novel inhibitor VE-822 results in selective sensitization of pancreatic tumors to radiation. Cell Death \& Disease, 2012. 3(12): p. e441-e441.

28. Tong, A.-J., et al., A Stringent Systems Approach Uncovers Gene-Specific Mechanisms Regulating Inflammation. Cell, 2016. 165(1): p. 165-179.

29. Cataldi, M., et al., Breaking resistance of pancreatic cancer cells to an attenuated vesicular stomatitis virus through a novel activity of IKK inhibitor TPCA-1. Virology, 2015. 485: p. 340-354.

30. Podolin, P.L., et al., Attenuation of Murine Collagen-Induced Arthritis by a Novel, Potent, Selective Small Molecule Inhibitor of I $\kappa$ B Kinase 2, TPCA-1 (2[(Aminocarbonyl)amino]-5-(4-fluorophenyl)-3-thiophenecarboxamide), Occurs via Reduction of Proinflammatory Cytokines and Antigen-Induced T Cell Proliferation. Journal of Pharmacology and Experimental Therapeutics, 2005. 312(1): p. 373.

31. Bartek, J. and J. Lukas, Chk1 and Chk2 kinases in checkpoint control and cancer. Cancer Cell, 2003. 3(5): p. 421-429.

32. Schmitt, Adam M., et al., p50 (NF- $\kappa B 1)$ Is an Effector Protein in the Cytotoxic Response to DNA Methylation Damage. Molecular Cell, 2011. 44(5): p. 785-796.

33. King, C., et al., Characterization and preclinical development of LY2603618: a selective and potent Chk1 inhibitor. Investigational New Drugs, 2014. 32(2): p. 213226. 
34. Norbury, C.J. and B. Zhivotovsky, DNA damage-induced apoptosis. Oncogene, 2004. 23(16): p. 2797-2808.

35. Sistigu, A., et al., Cancer cell-autonomous contribution of type I interferon signaling to the efficacy of chemotherapy. Nature Medicine, 2014. 20: p. 1301.

36. Balkwill, F., D. Watling, and J. Taylor-Papadimitriou, Inhibition by lymphoblastoid interferon of growth of cells derived from the human breast. International Journal of Cancer, 1978. 22(3): p. 258-265.

37. Widau, R.C., et al., RIG-I-like receptor LGP2 protects tumor cells from ionizing radiation. Proceedings of the National Academy of Sciences, 2014. 111(4): p. E484.

38. Chiappinelli, Katherine B., et al., Inhibiting DNA Methylation Causes an Interferon Response in Cancer via dsRNA Including Endogenous Retroviruses. Cell, 2015. 162(5): p. $974-986$.

39. Guo, Q., et al., STING promotes senescence, apoptosis, and extracellular matrix degradation in osteoarthritis via the $N F-\kappa B$ signaling pathway. Cell Death \& Disease, 2021. 12(1): p. 13.

40. Harberts, E., et al., MyD88 mediates the decision to die by apoptosis or necroptosis after UV irradiation. Innate Immunity, 2013. 20(5): p. 529-539.

41. Huang, T.T., et al., Sequential Modification of NEMO/IKK $\gamma$ by SUMO-1 and Ubiquitin Mediates NF- $\kappa B$ Activation by Genotoxic Stress. Cell, 2003. 115(5): p. 565-576.

42. Wang, P., et al., PUMA is directly activated by $N F-\kappa B$ and contributes to TNF- $\alpha$ induced apoptosis. Cell Death \& Differentiation, 2009. 16(9): p. 1192-1202.

43. Gatz, S.A. and L. Wiesmüller, p53 in recombination and repair. Cell Death \& Differentiation, 2006. 13(6): p. 1003-1016.

44. Boege, Y., et al., A Dual Role of Caspase-8 in Triggering and Sensing ProliferationAssociated DNA Damage, a Key Determinant of Liver Cancer Development. Cancer Cell, 2017. 32: p. 342 - 359.e10.

45. Feng, $\mathrm{H}$., et al., Interferon regulatory factor 1 (IRF1) and anti-pathogen innate immune responses. PLOS Pathogens, 2021. 17(1): p. e1009220.

46. Kim, W., et al., PIM1-Activated PRAS40 Regulates Radioresistance in Non-small Cell Lung Cancer Cells through Interplay with FOXO3a, 14-3-3 and Protein Phosphatases. Radiation Research, 2011. 176(5): p. 539-552.

47. Yang, H.J., et al., Investigation of Radiation-induced Transcriptome Profile of Radioresistant Non-small Cell Lung Cancer A549 Cells Using RNA-seq. PLOS ONE, 2013. 8(3): p. e59319. 
48. Pawlik, A., et al., Transcriptome Characterization Uncovers the Molecular Response of Hematopoietic Cells to Ionizing Radiation. Radiation Research, 2010. 175(1): p. 6682.

49. Zhang, Q., et al., Integrating radiosensitive genes improves prediction of radiosensitivity or radioresistance in patients with oesophageal cancer. Oncology letters, 2019. 17(6): p. 5377-5388.

50. Jones, K.B., et al., miRNA signatures associate with pathogenesis and progression of osteosarcoma. Cancer research, 2012. 72(7): p. 1865-1877.

51. Negishi, H., et al., Evidence for licensing of IFN- $\gamma$-induced IFN regulatory factor 1 transcription factor by MyD88 in Toll-like receptor-dependent gene induction program. Proceedings of the National Academy of Sciences, 2006. 103(41): p. 15136.

52. Wang, J., et al., IRF1 Promotes the Innate Immune Response to Viral Infection by Enhancing the Activation of IRF3. Journal of Virology, 2020. 94(22): p. e01231-20.

53. Bouker, K.B., et al., Interferon regulatory factor-1 (IRF-1) exhibits tumor suppressor activities in breast cancer associated with caspase activation and induction of apoptosis. Carcinogenesis, 2005. 26(9): p. 1527-1535.

54. Kim, P.K.M., et al., IRF-1 expression induces apoptosis and inhibits tumor growth in mouse mammary cancer cells in vitro and in vivo. Oncogene, 2004. 23(5): p. 11251135.

55. Tan, L., et al., Interferon regulatory factor-1 suppresses DNA damage response and reverses chemotherapy resistance by downregulating the expression of RAD51 in gastric cancer. Am J Cancer Res, 2020. 10(4): p. 1255-1270.

56. Thyrell, L., et al., Mechanisms of Interferon-alpha induced apoptosis in malignant cells. Oncogene, 2002. 21(8): p. 1251-1262.

57. Choi, E.A., et al., Stat1-dependent Induction of Tumor Necrosis Factor-related Apoptosis-inducing Ligand and the Cell-Surface Death Signaling Pathway by Interferon $\beta$ in Human Cancer Cells. Cancer Research, 2003. 63(17): p. 5299.

58. Kok, F., et al., Disentangling molecular mechanisms regulating sensitization of interferon alpha signal transduction. Molecular systems biology, 2020. 16(7): p. e8955-e8955.

59. Nan, J., et al., TPCA-1 Is a Direct Dual Inhibitor of STAT3 and NF- $\kappa B$ and Regresses Mutant EGFR-Associated Human Non-Small Cell Lung Cancers. Molecular Cancer Therapeutics, 2014. 13(3): p. 617. 
bioRxiv preprint doi: https://doi.org/10.1101/2021.10.21.465312; this version posted October 22,2021 . The copyright holder for this preprint (which was not certified by peer review) is the author/funder, who has granted bioRxiv a license to display the preprint in perpetuity. It is made available under aCC-BY-NC-ND 4.0 International license.

\section{Acknowledgements}

618 We want to thank Joschka Willemsen and Leanne Strauß for having started our lab's research line on

619 RLR-related cell death and providing valuable preliminary data, Hendrik Welsch for help with the 620 IncuCyte instrument and fruitful discussions, Volker Lohmann and Charlie Rice for provision of cell 621 lines, and Ralf Bartenschlager for providing an excellent research environment and supervising the 622 doctoral thesis of D.Z.. We are grateful to Eva Schnober, Hartmut Hengel and the rest of the team for 623 managing the fantastic Integrated Research Training Group "Immunovirology" as part of TRR179, 624 supporting D.Z. with a doctoral fellowship.

\section{Conflicts of interest}

626 The authors declare no conflict of interest.

\section{Author contributions}

628 This study was conceived and designed by M.B. and D.Z., critical intellectual input was provided by 629 V.G.M., experiments were performed by D.Z. with assistance and contributions by S.W., S.S.B. and 630 M.G.V., data was evaluated by D.Z. and M.B., the manuscript was written by D.Z. and M.B. and edited and approved of by all authors.

\section{Ethics Approval}

633 This research did not involve human or animal material; ethical approval was not required.

\section{$634 \quad$ Funding}

635 This research was funded by the German Research Foundation (Deutsche Forschungsgemeinschaft, 636 DFG), project BI1693/1-2 and project 272983813 CRC-TR 179 (TP11).

\section{Data Availability}

638 The raw data acquired for this study are available from the corresponding author on reasonable request. 